\title{
HIV-Positive Status Disclosure and Associated Factors among HIV-Positive Adult Patients Attending Art Clinics at Public Health Facilities of Butajira Town, Southern Ethiopia
}

\author{
Tamirat Melis Berhe $\mathbb{D}^{1},{ }^{1}$ Lire Lemma, ${ }^{1}$ Addissu Alemayehu, Desalegn Ajema, ${ }^{2}$ \\ Mustefa Glagn, ${ }^{2}$ and Samuel Dessu $\mathbb{1}^{3}$ \\ ${ }^{1}$ Wachamo University, College of Medicine and Health Science, Department of Public Health, Hosanna, Ethiopia \\ ${ }^{2}$ Arba Minch University, College of Medicine \& Health Sciences, Department of Public Health, Arba Minch, Ethiopia \\ ${ }^{3}$ Wolkite University, College of Medicine \& Health Science, Department of Public Health, Wolkite, Ethiopia \\ Correspondence should be addressed to Tamirat Melis Berhe; tamiraatmelis27@gmail.com
}

Received 20 February 2020; Revised 18 September 2020; Accepted 20 October 2020; Published 6 November 2020

Academic Editor: Bhaskaran Unnikrishnan

\begin{abstract}
Copyright (C) 2020 Tamirat Melis Berhe et al. This is an open access article distributed under the Creative Commons Attribution License, which permits unrestricted use, distribution, and reproduction in any medium, provided the original work is properly cited.
\end{abstract}

\begin{abstract}
Background. Human immunodeficiency virus-positive status disclosure is the process of informing one's HIV-positive status to others. It is the base for accessing care and treatment programs, attaining psychosocial support, reducing stigma, adhering to treatment, and promoting safer health. Even though different strategies were done in Ethiopia to increase the magnitude of HIV status disclosure among HIV-positive patients, the magnitude is still low. The magnitude of HIV-positive status disclosure was not assessed yet after initiation of the new strategy (test and treat strategy). The aim of this study is to assess the magnitude and factors associated with HIV-positive status disclosure among HIV-positive adults attending antiretroviral therapy clinic at the public health facilities of Butajira town. Methods. Institution-based cross-sectional study was conducted at public health facilities of Butajira town. A total of 414 study participants were selected by systematic random sampling technique. Data were collected by using pretested interviewer-administered semistructured questionnaire. The collected data were entered into EpiData3.1 and exported to SPSS version 23. Bivariate and multivariable logistic regression analysis was used to identify factors associated with HIV-positive status disclosure. The strength of association was assessed by crude odds ratio and adjusted odds ratio for bivariate and multivariable logistic regression analysis, respectively. Statistically significance was declared at $p$ value $<0.05$ and $95 \%$ CI. Results. The magnitude of HIV-positive status disclosure was 90\%. Discussing about safer sex (AOR: 3.5; 95\% CI: 1.3, 9.4), viral load suppression (AOR: 4; 95\% CI: 1.5, 10.1), having good ART adherence (AOR: 6; 95\% CI: 2.4, 14.0), receiving counseling (AOR: 2.5; 95\% CI: 1.01, 6.3), and perceiving stigma (AOR: 0.25; 95\% CI: 0.09, 0.60) were the independent factors associated with HIVpositive status disclosure. Conclusion. Although the majority of the participants (90\%) of them disclosed their HIV-positive status, lack of disclosure by few people can tackle HIV prevention and control programs. Health programs could improve disclosure of HIV-positive status by providing counseling service, strengthening adherence of antiretroviral therapy, suppressing viral load, and avoiding (reducing) stigma on HIV-positive patients by their community.
\end{abstract}

\section{Background}

Since the starting of HIV epidemic, above 70 million people have been infected with HIV and 35 million people died worldwide. There had been 36.9 million people having HIV in their blood. Africa (WHO region) is one of the HIV prevalent regions, with $4.1 \%$ of the population living with
HIV and accounts for $66 \%$ of people living with HIV at the end of 2017 [1]. Ethiopia has a large and very vulnerable population that there were more than half million $(729,089)$ HIV-positive population, 21,606 new HIV infections, and 10,960 deaths in 2018 [2].

HIV-positive status disclosure refers to the act of informing the HIV status of an infected person with or 
without consent to other people [3]. Disclosure of HIVpositive status plays an important role in strengthening ART adherence and prevention of HIV $[4,5]$. It is useful for both the infected individual and the community. It inspires people to seek HIV testing and counseling, change behavior, and decrease the prevalence of HIV [6].

The magnitude of HIV-positive status disclosure in developing countries is lower than in developed countries. The magnitude of HIV-positive status disclosure in developing countries ranged from $16.7 \%$ to $86 \%$ with the average of $49 \%$. This indicates $50 \%$ of people living with HIV/AIDS in developing countries disclosed their HIV-positive status to other person. But, in developed countries, the average magnitude of HIV-positive disclosure status was 79\% [7].

The disclosure level of $56.3 \%$ was documented by the study conducted at the Mityanaa district hospital of Uganda [8]. According to the study conducted in Southwest Ethiopia; of the total respondents, $37.6 \%$ had disclosed their HIV-positive status in general. The failure of people infected with HIV to disclose their HIV-positive status can expose their sexual partner and other relatives that have close contact with them to be infected by the virus [9]. Nondisclosure of HIV-positive status to someone has many consequences like distress, loneliness and social isolation [10], lack of support, infection from their partners with a new type of HIV strain [11], refuse to initiate ART, poor adherence of ART, poor utilization of condom, and chance of mother-tochild transmission of HIV [7]. The aim of this study was to assess the magnitude and factors associated with HIVpositive status disclosure among ART patients attending public health facilities of Butajira town, Southern Ethiopia, 2019.

\section{Methods}

2.1. Study Setting and Design. This study was conducted at public health facilities of Butajira town, Gurage Zone, Sothern Ethiopia, $135 \mathrm{~km}$ south from Addis Ababa, located at the base of the Zebidar massive in the Gurage Zone of the Southern Nations, Nationalities and Peoples' Region. The town has an estimated total population of 50,290 (24,642 males and 25,648 females). The town has one general hospital, one private hospital, one health center, ten private clinics, and five health posts. ART services provided both at Butajira General Hospital and Butajira Health Center with average monthly attendant of 1021 ART follow-up patients. An institution-based cross-sectional study was conducted from March 01, 2019, to March 30, 2019, on ART clients. The study populations were all HIV-positive adult patients who attending ART clinic for ART treatment at public health facilities of Butajira town.

2.2. Sample Size and Sampling Procedure. The sample size was determined by using a single population proportion formula by considering the following assumptions: $95 \% \mathrm{CI}$, $5 \%$ margin of error, and magnitude of HIV-positive status disclosure in Jimma Specialized Hospital, Ethiopia, (p) which is $37.6 \%$ [12]. Accordingly, the calculated sample size with $15 \%$ nonresponse rate for this study was 414 . The total number of adults who were on ART at the public health facilities of Butajira town was 945 ( 813 from Butajira General Hospital and 132 from Butajira Health Center). The sample size was allocated proportionally for the hospital and health center and selected by using systematic random sampling. Since total sample size for this study was $414, k=945 /$ $414=2.28=2$. Lottery method was used to determine every $1^{\text {st }}$ or the $2^{\text {nd }}$ case. Therefore, every $1^{\text {st }}$ client from the first two patients who came for ART service was selected systematically as the study participants.

\subsection{Operational Definition}

2.3.1. HIV-Positive Status Disclosure. Informing one's HIVpositive status to another person (sexual partner, families or friends, or others) [12].

2.3.2. Current Viral Load Suppression. The last (recent) viral load tested was less than 1000 viral copies per milliliter of blood sample [13].

2.4. Data Collection Tools and Procedure. The standardized data collection tool was developed through reviewing of related literature [14-16]. It contains information on sociodemographic factors, individual factors, and disease-related factors. Perceived stigma was assessed by the stigma assessment tool which contains 10 stigma assessment questions.

First, the questionnaire was prepared in English, translated to Amharic and then back to English. A week before the beginning of actual data collection, a pretest was done on $5 \%$ of the sample size in Agena town. The data were collected by six case managers/adherence supporters and supervised by two public health officers who were trained on HIV/AIDS comprehensive management and care. Training was given for data collectors and supervisors on how to manage the data collection process. Those data collectors and supervisors who work at Butajira General Hospital were assigned as data collectors of Butajira Health Center and vice versa.

The data were collected by face-to-face interviews and reviewing patient cards by using interviewer-administered semistructured questionnaire.

2.5. Data Processing and Analysis. Data were entered into Epidata version 3.1 and then exported to SPSS version 23 for further analysis. The descriptive analysis like percentage, frequency, and mean was calculated. Bivariate and multivariable logistic regression analysis was used to identify associations between dependent and independent variables. In bivariate analysis, variables with $p \leq 0.25$ were entered into multivariable logistic regression analysis. The Hosmer-Lemeshow test was used to check the appropriateness of the model for analysis. The possible effects of confounders were controlled through multivariable logistic regression analysis. The association between the explanatory and 
dependent variables were assessed at the $p$ value of 0.05 . The variables that show $p$ value $<0.05$ were declared as statistically significant variables in multivariable logistic regression analysis. The degree of association between independent and dependent variables was assessed using crude odds ratio (COR) and adjusted odds ratio (AOR) for bivariate and multivariable logistic regression, respectively, with $95 \%$ confidence interval.

\section{Results}

3.1. Sociodemographic Characteristics. A total of 405 ART patients participated in this study were giving the response rate of $97.8 \%$. The age of respondents range from 19 to 70 years with the mean age of $38 \pm 10$ years. Of the total participants, 274 (67.7\%) were females. Majority $323(79.8 \%)$ of respondents were from urban areas. About 249 (61\%) of the study participants were from Gurage ethnic group. More than half $(216,53.3 \%)$ of the respondents were Orthodox Christian. One hundred sixty five $(37.7 \%)$ did not attend formal education. Two hundred ten of the respondents (51.9\%) were married (see Table 1).

3.2. Disease-Related Characteristics of Study Participants. From a total of 393 respondents who had tested for current viral load, $313(80 \%)$ had current suppressed viral load $(<1000$ copies/ml) and 213 (59\%) had low CD4 count at ART start. Majority $(331,82 \%)$ of the respondents had good ART adherence (see Table 2).

3.3. Individual Characteristics of Study Participants. From a total of 405 respondents, 303 (75\%) had sexual partners. Among those who had sexual partners, 279 (92\%) know their sexual partner status (see Table 3)

3.4. Magnitude of HIV-Positive Status Disclosure. Among the study participants, $90 \%$ of them disclosed their HIV-positive status (see Figure 1).

\subsection{Factors Associated With HIV-Positive Status Disclosure.}

Bivariate analysis was performed to assess factors which were significantly associated with HIV-positive status disclosure. The bivariate analysis showed that discussing about safer sex, viral load suppression, WHO stage at ART start, ART adherence, presence of comorbid condition, receiving counseling, perceived stigma, CD4 count at ART start, current CD4 count, general health condition of the patient, having sexual partner, and belonging to the HIV/AIDS support group were variables which were a candidate for multivariable binary logistic regression analysis.

In multivariable logistic regression analysis, discuss about safer sex, viral load suppression, having good ART adherence, receiving counseling service about HIV-positive status disclosure, and perceived stigma were factors significantly associated with HIV-positive status disclosure. The odds of HIV-positive status disclosure in ART patients who had a free discussion about safer sex were 3.5 times
TABLE 1: Sociodemographic characteristics of ART patients attending public health facilities of Butajira town, Southern Ethiopia, $2019(n=405)$.

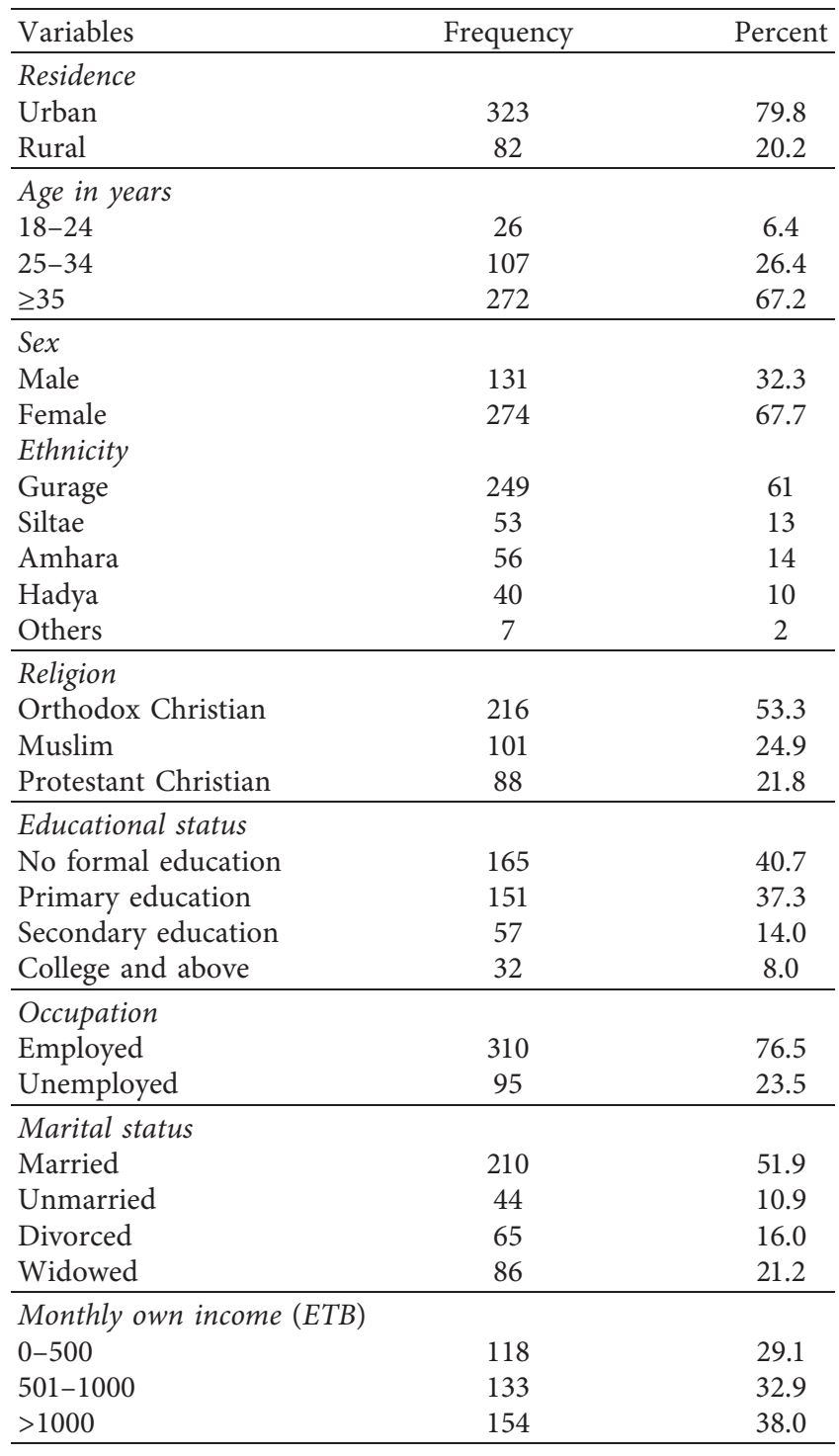

more likely to disclose their HIV-positive status than their counterparts (AOR: 3.5; 95\% CI: 1.3, 9.4). ART patients who had good ART adherence were 6 times more likely to disclose their HIV-positive status than those who had poor ART adherence (AOR: 5.9; 95\% CI: 2.4, 14). Being in HIV viral load suppression is 4 times more likely to disclose HIVpositive status than being in HIV viral load non suppression (AOR: 3.9; 95\% CI: 1.5, 10). Study participants those who perceived stigma were $75 \%$ times less likely to disclose their HIV-positive status than their counterparts (AOR: 0.25; 95\% CI: $0.10,0.6)$ (Table 4).

\section{Discussion}

This study had assessed the magnitude of HIV-positive status disclosure and factors associated with it among people living with HIV/AIDS attending ART clinic in the public 
TABLE 2: Individual and disease-related factors of ART patients attending public health facilities of Butajira town, Southern Ethiopia, 2019 $(n=405)$.

\begin{tabular}{|c|c|c|c|}
\hline Variables & Categories & Frequency & Percent \\
\hline \multirow{2}{*}{ CD4 count at ART start (cells/ml) } & $<250$ & 213 & 59 \\
\hline & $\geq 250$ & 148 & 41 \\
\hline \multirow{2}{*}{ Current CD4 count (cells/ml) } & $<250$ & 31 & 8 \\
\hline & $\geq 250$ & 330 & 92 \\
\hline \multirow{2}{*}{ Current viral load (copies/ml) } & $<1000$ & 313 & 80 \\
\hline & $\geq 1000$ & 80 & 20 \\
\hline \multirow{2}{*}{ WHO stage at ART start } & I-II & 268 & 66 \\
\hline & III-IV & 137 & 34 \\
\hline \multirow{2}{*}{ Current WHO stage } & I-II & 380 & 94 \\
\hline & III-IV & 25 & 6 \\
\hline \multirow{3}{*}{ Duration of ART treatment started (in years) } & $<1$ & 78 & 19 \\
\hline & $1-2$ & 42 & 10 \\
\hline & $>2$ & 285 & 71 \\
\hline \multirow{2}{*}{ General health } & Worked & 391 & 97 \\
\hline & Ambulatory & 14 & 3 \\
\hline \multirow{2}{*}{ ART adherence } & Good & 331 & 82 \\
\hline & Poor & 74 & 18 \\
\hline
\end{tabular}

TABLE 3: Individual and psychosocial factors of adult ART patients attending public health facilities of Butajira town, Southern Ethiopia, $2019(n=405)$.

\begin{tabular}{|c|c|c|c|}
\hline Variables & Categories & Frequency & Percent \\
\hline \multirow{2}{*}{ Have sexual partner } & Yes & 303 & 75 \\
\hline & No & 102 & 25 \\
\hline \multirow{2}{*}{ Know status of sexual partner } & Yes & 279 & 92 \\
\hline & No & 24 & 8 \\
\hline \multirow{2}{*}{ Status of sexual partner } & Positive & 237 & 85 \\
\hline & Negative & 42 & 15 \\
\hline \multirow{2}{*}{ Belongs to support group } & Yes & 98 & 24 \\
\hline & No & 307 & 76 \\
\hline \multirow{2}{*}{ Discuss about safer sex } & Yes & 247 & 60 \\
\hline & No & 158 & 40 \\
\hline \multirow{2}{*}{ Receive counselling } & Yes & 254 & 63 \\
\hline & No & 151 & 27 \\
\hline \multirow{2}{*}{ Have knowledge on importance of disclosure } & Yes & 273 & 58.5 \\
\hline & No & 168 & 41.5 \\
\hline \multirow{2}{*}{ Perceived stigma } & Yes & 116 & 29 \\
\hline & No & 289 & 71 \\
\hline \multirow{2}{*}{ Perceived discrimination } & Yes & 27 & 7 \\
\hline & No & 378 & 93 \\
\hline \multirow{2}{*}{ History of sexual intercourse with in previous six month } & Yes & 312 & 77 \\
\hline & No & 93 & 23 \\
\hline \multirow{2}{*}{ Utilize condom } & Yes & 253 & 81 \\
\hline & No & 59 & 19 \\
\hline
\end{tabular}

health facilities of Butajira town, Gurage Zone, Southern Ethiopia. From the total respondents, 365 (90\%) have disclosed their HIV-positive status to another person.

This finding is lower than studies conducted in National Hospital of Abuja, Nigeria (95\%), and Northeastern Nigeria $(97.5 \%)[17,18]$. This might be because of the difference in economic status, culture, and ART service delivery programs in Ethiopia and Nigeria. The difference might also be attributed to there were support group service providers at
ART centers for HIV-positive patients who were enrolled in HIV $\backslash$ AIDS care and treatment center of Nigeria.

But this finding is higher than studies conducted in Jima Specialized Hospital, Ethiopia (37.6\%), Axum Health Facilities, Ethiopia (80.1\%), and Ambo Hospital, Ethiopia $(86.2 \%)[12,16,19]$. The possible explanation for this difference might be because of the collaboration work of governmental and nongovernmental organizations working in public health facilities of Butajira town. There were four 


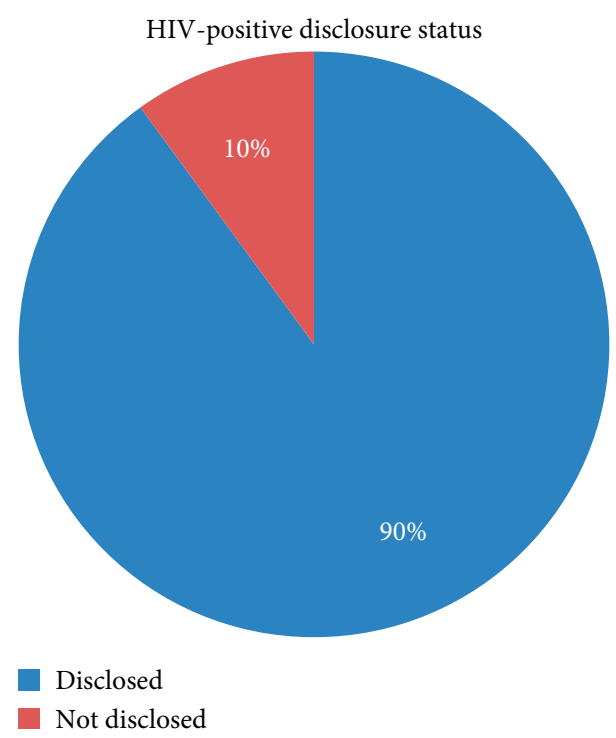

FIGURE 1: -Magnitude of HIV-positive status disclosure among adult ART patients attending ART clinics at public health facilities of Butajira town, Southern Ethiopia, $2019(n=405)$.

TABLE 4: Multivariable logistic regression analysis for factors associated with HIV-positive status disclosure among ART patients attending ART clinic in public health facilities of Butajira town, Southern Ethiopia, $2019(n=405)$.

\begin{tabular}{|c|c|c|c|c|}
\hline \multirow[t]{2}{*}{ Variables } & \multicolumn{2}{|c|}{$\begin{array}{l}\text { Variables disclose } \\
\text { HIV-positive status }\end{array}$} & \multirow[t]{2}{*}{ COR $(95 \%$ CI $)$} & \multirow[t]{2}{*}{ AOR (95\% CI) } \\
\hline & Yes & No & & \\
\hline \multicolumn{5}{|c|}{ Discuss about safer sex } \\
\hline Yes & 235 & 12 & $4(2.07,8.57)^{* *}$ & $3.5(1.34,9.45)^{* *}$ \\
\hline No & 130 & 28 & 1 & 1 \\
\hline \multicolumn{5}{|l|}{ Viral load } \\
\hline Suppressed & 290 & 23 & $3.4(1.71,6.74)^{* *}$ & $3.9(1.52,10.11)^{* *}$ \\
\hline Not suppressed & 63 & 17 & 1 & 1 \\
\hline \multicolumn{5}{|l|}{ ART adherence } \\
\hline Good & 307 & 24 & $3.5(1.76,7.05)^{* *}$ & $5.9(2.41,14.54)^{* *}$ \\
\hline Poor & 58 & 16 & 1 & 1 \\
\hline \multicolumn{5}{|c|}{ Receive counselling } \\
\hline Yes & 239 & 15 & $3.1(1.60,6.212)^{* *}$ & $2.5(1.01,6.30)^{*}$ \\
\hline No & 126 & 25 & 1 & 1 \\
\hline \multicolumn{5}{|l|}{ Perceived stigma } \\
\hline Yes & 93 & 23 & $0.25(.13, .49)^{* * *}$ & $0.25(0.10,0.63)^{* *}$ \\
\hline No & 272 & 17 & 1 & 1 \\
\hline
\end{tabular}

nongovernmental projects which work on activities like providing counseling service, partner notification, family index case finding, and adhering the patient for ART service. Four NGOS working in one project might be the justification for this difference. Study time difference might be the other possible explanation. The difference might also be attributed to the initiation of a new program (test and treat strategy).

This study revealed that receiving counseling about HIV/ AIDS causes more likely to disclose HIV-positive status than not receiving counseling about HIV-positive status disclosure. It is consistent with the study conducted at Bale Zone Hospitals, Ethiopia; Woldia Hospital, Ethiopia, and Mityana Hospital, Uganda, in which those who received counseling were more likely to disclose their HIV-positive status than those who did not receive counseling service about HIV status disclosure $[8,11,20]$. In another study conducted in Kisarawe Hospital, Tanzania, those who did not receive counseling on HIV/AIDS status disclosure were less likely to disclose their HIV-positive status than those who received HIV-positive status disclosure counseling [21]. The possible explanation might be due to the fact that receiving counseling about HIV/AIDS encourages disclosure, increase awareness, and also bring behavioral change about disclosure. As a result, individuals were able to overcome feelings of shame which facilitated disclosure of HIV-positive status because the patients might knew about the benefits of HIV- 
positive status disclosure. The other justification might be as patients started ART treatment immediately, they would had frequent contact with health professionals indeed will expose to receive counseling services from health professionals.

This study showed that those who had a discussion about safer sex were more likely to disclose their HIV-positive status than those that did not discuss about safer sex. It is consistent with a study conducted at Jimma town, Ethiopia, in which those who had a discussion about safer sex were more likely to disclose their HIV-positive status than their counterparts [22]. This might be due to the fact that discussion about safer sex may encourage them to have open communication and freedom to disclose their HIV-positive status.

This finding revealed that those who had good ART adherence were more likely to disclose their HIV-positive status than those who had poor ART adherence. This finding is consistent with a study conducted in Tigray, Ethiopia, and Sokodé Regional Hospital, Togo, in which those who had good ART adherence disclosed their HIV-positive status more likely compared to those who had poor ART adherence $[14,23]$. This might be due to the fact that adhering to ART might enhance adherence to service provided at health facilities (emphasized on HIV status disclosure), which in turn increases awareness on HIV-positive status disclosure.

This study showed that study participants who had viral load suppression were more likely to disclose their HIVpositive status than those who had a nonsuppressed viral load. It is consistent with the study conducted in the United Kingdom which showed that those who had suppressed viral load were more likely to disclose their HIV-positive status than those who had a nonsuppressed viral load. This might be due to the fact that those who had suppressed viral load had a good adherence and contact with health professionals frequently [24].

This study revealed that ART patients that perceived stigma were less likely to disclose their HIV-positive status than those who perceived stigma. It is consistent with a study conducted in Sydney, Australia, in which those who perceived stigma were less likely to disclose their HIV status than their counterparts [25]. The possible justification might be that the stigmatized patients might fear consequences of disclosure like depression, social withdrawal, psychological stress, and loss of support from families.

\section{Limitations of the Study}

This study was based on self-reporting of the disclosure status which might overestimate the outcome variable because of social desirability bias. The finding may not be inferences to the general population since it was facilitybased study. The cross-sectional study design has weaknesses in establishing temporal relationship. The wealth index was not assessed.

\section{Conclusion}

The magnitude of HIV-positive status disclosure in public health facilities of Butajira town is good. Receiving counseling on HIV-positive status disclosure, free discussion about safer sex, viral load suppression, having good ART adherence, and perceiving stigma are independent factors associated with HIV-positive status disclosure.

\section{Abbreviations \\ AOR: Adjusted odds ratio \\ HIV: Human immunodeficiency virus \\ ART: Antiretroviral therapy \\ AIDS: Acquired immunodeficiency syndrome.}

\section{Data Availability}

The data sets used/or analyzed during the current study are available from the corresponding author on reasonable request.

\section{Ethical Approval}

Ethical clearance was obtained from Institutional Ethical Review Board, College of Medicine and Health Science, Arba Minch University. By using the support letter which was approved by from Arba Minch University Institutional Ethical Review Board, Department of Public Health, permission to conduct the research was obtained from Gurage Zone Health Office, Butajira General Hospital, and Butajira Health Center.

\section{Consent}

Verbal consent was obtained from study participants to assure the voluntariness of the participants. Respondents were informed about the purpose and procedure of the study, the importance of their participation, the right to withdraw at any time if they want, and about privacy and confidentiality of the information given by each respondent. The information was kept properly, and the name was not recorded.

\section{Conflicts of Interest}

The authors declare that they have no conflicts of interest.

\section{Authors' Contributions}

TM designed the study, was involved in data collection, done analysis, and interpretation of the result, drafted the paper, and participated in preparing all versions of the manuscript. AA, DA, SD, MG, and LL assisted in the design and the proposal development, monitored data collection, assisted during analysis, and revised subsequent drafts of the paper. All authors read and approved the final manuscript.

\section{Acknowledgments}

The authors would like to express their heartfelt appreciation to data collectors and all ART patients who were involved as study participants in this study. They are also grateful to all 
their colleagues and health professionals who supported the study process.

\section{References}

[1] WHO, Global Health Observatory Data on Prevalence of HIV Among Adult Age 15-49, WHO, Geneva, Switzerland, 2017, https://www.who.int/hiv/data/en/.

[2] HAPCO/EPHI, HIV epidemic estimates 2017-2021, Ethiopia A. A: EPHI, 2017.

[3] Prevention in focus spotlight in programing and research spring 2016 jump to navigation jump to contents $2016 \mathrm{https} / / /$ www.catie.ca/en/pif/spring-2016/disclosure-programmingreview-evidence.

[4] T. Gadisa, O. Tymejczyk, S. G. Kulkarni et al., "Disclosure history among persons initiating antiretroviral treatment at six HIV clinics in Oromia, Ethiopia, 2012-2013," AIDS and Behavior, vol. 21, no. 1, pp. 70-81, 2017.

[5] C. L. Mattson, M. Freedman, J. L. Beer, P. Sullivan, and J. Skarbinsk, "Prevalence and predictors of HIV disclosure among adults receiving care in the United States," 2019.

[6] S. Maman and A. Medley, "Gender dimensions of HIV status disclosure to sexual partners: rates barriers and outcomes," 2004.

[7] WHO, Geneva, Switzerland, WHO, HIV Status Disclosure to Sexual Partners: Rates, Barriers and Outcomes for Women, 2004.

[8] I. Kadowa and F. Nuwaha, "Factors influencing disclosure of HIV positive status in Mityana district of Uganda," African health sciences, vol. 9, no. 1, pp. 26-33, 2009.

[9] M. Genet, G. Sebsibie, and T. Gultie, "Disclosure of HIV seropositive status to sexual partners and its associated factors among patients attending antiretroviral treatment clinic follow up at Mekelle Hospital, Ethiopia: A cross sectional study," BMC Research Notes, vol. 8, no. 1, p. 109, 2015.

[10] B. D. Winters, S. J. Weaver, E. R. Pfoh, T. Yang, J. C. Pham, and S. M. Dy, "Rapid-response systems as a patient safety strategy," Annals of Internal Medicine, vol. 158, no. 5, pp. 417-425, 2013.

[11] T. D. Geremew, R. A. Nuri, and J. K. Esmael, "Sero status disclosure to sexual partner and associated factors among adult HIV positive patients in Bale zone hospitals, Oromia region, Ethiopia: Institution based cross-sectional study," Open Journal of Epidemiology, vol. 8, no. 2, p. 43, 2018.

[12] T. Tesfaye, J. Darega, T. Belachew, and A. Abera, "HIV positive sero-status disclosure and its determinants among people living with HIV/AIDS following ART clinic in Jimma University specialized hospital, Southwest Ethiopia: A facilitybased cross-sectional study," Archives of Public Health, vol. 76, no. 1, p. 1, 2018.

[13] J. Thorman, P. Björkman, F. Tesfaye, A. Jeylan, T. T. Balcha, and A. Reepalu, "Validation of the Viral Load Testing Criteria - an algorithm for targeted viral load testing in HIV-positive adults receiving antiretroviral therapy," Tropical Medicine \& International Health, vol. 24, no. 3, pp. 356-362, 2019.

[14] I. Yaya, B. Saka, D. E. Landoh et al., "HIV status disclosure to sexual partners, among people living with HIV and AIDS on antiretroviral therapy at Sokodé regional hospital," Togo. PLoS One, vol. 10, no. 2, Article ID e0118157, 2015.

[15] Konam Kab barriers to disclosure of HIV positive status to sexual partner(s), 2015.

[16] S. Fituma, "HIV positive status disclosure and highly active antiretroviral therapy adherence among people living with HIV in Ambo Hospital, West Shewa Zone, Oromia Region,
Ethiopia," Obstetrics \& Gynecology International Journal, vol. 5, no. 2, 2016.

[17] R. S. Dankoli, A. A. Aliyu, P. Nsubuga et al., "HIV disclosure status and factors among adult HIV positive patients in a secondary health facility in North-Eastern Nigeria, 2011," The Pan African Medical Journal, vol. 18, no. Suppl 1, 2014.

[18] O. F. Martins, H. C. Ngong, I. S. Dongs, and K. C. Ngong, "Rates, factors, timing and outcomes of HIV status disclosure among patients attending the special treatment clinic of the national hospital Abuja Nigeria," International Journal of HIV/AIDS Prevention, Education, and Behavioural Science, vol. 2, no. 3, pp. 13-19, 2016.

[19] H. B. Alema, W. A. Yalew, M. B. Beyene, and M. G. Woldu, "HIV positive status disclosure and associated factors among HIV positive adults in Axum health facilities, Tigray, Northern Ethiopia," Science Journal of Public Health, vol. 3, pp. 61-66, 2015.

[20] T. A. Erku, B. Megabiaw, and M. Wubshet, "Predictors of HIV status disclosure to sexual partners among people living with HIV/AIDS in Ethiopia," The Pan African Medical Journal, vol. 13, 2012.

[21] J. A. Mwanga, HIV Sero Status Disclosure and Associated Factors Among People Living with HIV/AIDS Attending a Care and Treatment Centre in Kisarawe District Hospital, Muhimbili University of Health and Allied Sciences, Dar es Salaam, Tanzania, 2012

[22] G. Kassahun, Z. Tenaw, T. Belachew, and M. Sinaga, "Determinants and status of HIV disclosure among reproductive age women on antiretroviral therapy at three health facilities in Jimma town, Ethiopia," Health Science Journal, vol. 12, no. 2, 2017.

[23] H. Ebuy, H. Yebyo, and M. Alemayehu, "Level of adherence and predictors of adherence to the Option B+ PMTCT programme in Tigray, Northern Ethiopia," International Journal of Infectious Diseases, vol. 33, pp. 123-129, 2015.

[24] M. Daskalopoulou, F. C. Lampe, F. C. Lampe et al., "Nondisclosure of HIV status and associations with psychological factors, ART non-adherence, and viral load non-suppression among people living with HIV in the UK," AIDS and Behavior, vol. 21, no. 1, pp. 184-195, 2017.

[25] H. Hosseinzadeh, S. Z. Hossain, and S. Bazargan-Hejazi, "Perceived stigma and social risk of HIV testing and disclosure among Iranian-Australians living in the sydney metropolitan area," Sexual Health, vol. 9, no. 2, p. 171, 2011. 